\title{
LETTER
}

\section{An interaction switch predicts the nested architecture of mutualistic networks}

\author{
Feng Zhang, ${ }^{1}$ * Cang Hui ${ }^{1}$ and \\ John S. Terblanche ${ }^{2}$ \\ ${ }^{1}$ Centre for Invasion Biology, \\ Department of Botany and Zoology, \\ Stellenbosch University, Private \\ Bag X1, Matieland 7602, South \\ Africa \\ ${ }^{2}$ Department of Conservation \\ Ecology and Entomology, \\ Stellenbosch University, Private Bag \\ X1, Matieland 7602, South Africa \\ *Correspondence: E-mail: \\ fzhang@sun.ac.za
}

\begin{abstract}
Nested architecture is distinctive in plant-animal mutualistic networks. However, to date an integrative and quantitative explanation has been lacking. It is evident that species often switch their interactive partners in realworld mutualistic networks such as pollination and seed-dispersal networks. By incorporating an interaction switch into a novel multi-population model, we show that the nested architecture rapidly emerges from an initially random network. The model allowing interaction switches between partner species produced predictions which fit remarkably well with observations from 81 empirical networks. Thus, the nested architecture in mutualistic networks could be an intrinsic physical structure of dynamic networks and the interaction switch is likely a key ecological process that results in nestedness of real-world networks. Identifying the biological processes responsible for network structures is thus crucial for understanding the architecture of ecological networks.
\end{abstract}

\section{Keywords}

Abundance, adaptive behaviour, antagonistic network, dynamic network, functional response, morphological trait, nestedness, network size, pollination, seed disperser.

Ecology Letters (2011) 14: 797-803

\section{INTRODUCTION}

Mutualistic interactions are crucial processes to sustain ecosystem function and services, foster biodiversity and affect community stability (Bascompte \& Jordano 2007; Okuyama \& Holland 2008; Bastolla et al. 2009; Thébault \& Fontaine 2010). Specifically, bipartite networks have been applied to depict mutualistic interactions such as in pollination and seed-dispersal communities (Bascompte \& Jordano 2007). A distinctive feature of mutualistic networks is their nested architecture (but see Bascompte et al. 2003; Guimarães et al. 2006, 2007a; Chamberlain et al. 2010); that is, specialists only interact with a proper subset of the species interacting with the more generalist species (Bascompte et al. 2003; Ollerton et al. 2003; Nielsen \& Bascompte 2007). Although a neutral model only considering fixed species abundance has been proposed for explaining such nested network architecture (Krishna et al. 2008), the model considerably overestimated the observed nestedness. As multiple biological mechanisms could be responsible for the nested architecture (Vázquez et al. 2009), an integrative and quantitative understanding of this distinctive feature of mutualistic networks is still lacking.

Mounting evidence has suggested that species often switch their interaction partners not only in antagonistic interactions (i.e. food webs; Murdoch 1969; Staniczenko et al. 2010) but also in mutualistic networks (Basilio et al. 2006; Fortuna \& Bascompte 2006; Olesen et al. 2008; Petanidou et al. 2008). For instance, in a pollination network, pollinators continually switch the plant species with which they interact in response to environmental disturbances and the availability of resources (Whittall \& Hodges 2007), whereas plants can also adjust phenology (e.g. flowering time) and morphology (e.g. flower heterostyly) to affect their pollinators (Aizen \& Vázquez 2006; Barrett 2010; Kaiser-Bunbury et al. 2010). This implies a dynamic nature to ecological networks; i.e. both species abundance and species interaction could affect each other and change over time. Such interaction switches between species could not only have ecological and environmental reasons (e.g. resource availability) but also reflect an adaptive behaviour of species for enhancing the efficiency of resource utilisation. Compared with fixed interactions between species, an interaction switch (alternatively, the 'rewiring' of interactions between species) can lead to greater stability in food webs (Staniczenko et al. 2010) and pollination networks (Kaiser-Bunbury et al. 2010). As such, a dynamic network with switching interactions can be considered as a specific form of an adaptive network. Foraging adaptation (shifts in prey selection) in adaptive networks can predict the emergence of food-web complexity (Kondoh 2003; Beckerman et al. 2006; Garcia-Domingo \& Saldana 2007; Petchey et al. 2008). Because the consumer-resource interactions of mutualisms resemble a predator-prey interaction (Holland \& DeAngelis 2010), it is expected that such adaptive behaviour of an interaction switch could play a crucial role in determining the architecture of mutualistic networks.

Using a mathematical model for depicting population dynamics in mutualistic networks, we first introduce this interaction switch into the model and then investigate whether the distinctive nested architecture of mutualistic networks can be robustly predicted. Importantly, we are able to demonstrate that a mutualistic network converges from an initial random network to a nested network with an intrinsic stable level of nestedness. This model was well supported by several tests of the model's predictions with multiple observations from empirical pollination and seed-dispersal networks (see supplementary Section S1). We believe this model to be a significant conceptual contribution as it facilitates further research on the role of species abundance and/or morphology in forming the nested architecture of mutualistic networks and provides an explanation for how real-world networks self organize and develop. 


\section{MODEL AND METHODS}

Assume that a plant-animal mutualistic network consists of $m$ plant species and $n$ animal species. The population dynamics of plant $i$ and animal $j$ can thus be depicted by its own density-dependent process plus the functional response to its mutualistic interactions:

$$
\begin{aligned}
& \frac{1}{N_{i}^{(P)}} \frac{d N_{i}^{(P)}}{d t}=r_{i}^{(P)}-c_{i}^{(P)} N_{i}^{(P)}+\frac{\sum_{j} b_{i j}^{(P)} a_{i j} v_{i j} N_{j}^{(A)}}{1+\sum_{k} b a_{i k} v_{i k} N_{k}^{(A)}} \\
& \frac{1}{N_{j}^{(A)}} \frac{d N_{j}^{(A)}}{d t}=r_{j}^{(A)}-c_{j}^{(A)} N_{j}^{(A)}+\frac{\sum_{i} b_{j i}^{(A)} a_{i j} v_{i j} N_{i}^{(P)}}{1+\sum_{k} b a_{k j} v_{k j} N_{k}^{(P)}},
\end{aligned}
$$

where the superscripts $(P)$ and $(A)$ denote plants and animals respectively, $N_{i}$ the population size of species $i$, parameters $r_{i}$ and $c_{i}$ the intrinsic growth rate and density-dependent coefficient. The last term describes the Holling's (type II; 1959) functional response to the mutualistic interaction (see supplementary Section S2), where the interaction matrix $\left\{a_{i j}\right\}$ denotes whether plant $i$ and animal $j$ interact $\left(a_{i j}=1\right)$ or not $\left(a_{i j}=0\right)$; the preference matrix $\left\{v_{i j}\right\}$ describe the probability of an interaction once animal $j$ encounters plant $i$; the benefits matrix $\left\{b_{i j}\right\}$ describes the benefit of species $j$ to $i$; $b$ is the handling time (following Holland et al. (2006), we choose the same value for both plants and animals; $h=0.1$ as in Bastolla et al. (2009)). We ignored competition between plants or between animals following several previous studies (Bascompte et al. 2006; Holland et al. 2006; Okuyama \& Holland 2008; Thébault \& Fontaine 2010).

The numerical solutions for the above model were obtained via the Euler method with a time step of 0.01 starting from initial population sizes randomly assigned between 0 and 1 (Ascher \& Petzold 1998). In the calculation, values of intrinsic growth rates $\left(r_{i}\right)$, density-dependent coefficient $\left(c_{i}\right)$ and the benefits matrix $\left\{b_{i j}\right\}$ were randomly assigned numbers between 0 and 1 , and the number of interactions and network size $(m \times n)$ were assigned according to data from the real mutualistic network (see supplementary Section S1). The interaction matrix $\left\{a_{i j}\right\}$ was initialised by a random binary $(0 / 1)$ matrix but with a fixed number of interactions (i.e. $I=\sum_{i} \sum_{j} a_{i j}$ remains constant in the calculation) and elements in the preference matrix $\left\{v_{i j}\right\}$ were randomly assigned numbers between 0 and 1 . In each time step, one randomly chosen species stops the interaction of the minimum gain in per capita population growth rate [i.e. the interaction with the least $b_{i j} a_{i j} v_{i j} N_{j}$ in the denominator of the last term in eqn (1)]. To keep the number of interactions constant, a randomly chosen interaction with $a_{i j}=0$ is reassigned to 1 and then the preference value of this new interaction $\left(v_{i j}\right)$ is updated by a random number between 0 and 1 . This rule of an interaction switch, although simple, ensures a basic process of behavioural adaptation: individuals of each species try to effectively take their limited time to interact only with species that can provide a greater benefit rather than potentially wasting time on many lowbenefit interactions (as a consequence of the functional response; see supplementary Section S2; Holling 1959). Therefore, each species in the network can enhance its benefit through readjusting mutualistic interactions.

As switching interactive partners (i.e. the 'rule' of the interaction switch) depends on the partner's abundance and the benefits provided $\left(b_{i j} a_{i j} v_{i j} N_{j}\right)$, we further evaluated how species abundance and benefits contribute to the nested architecture by examining two special cases of this model: benefit-neutral and demography-neutral interactions. The former refers to no variation in both the benefits $\left(b_{i j}\right)$ and the preference $\left(v_{i j}\right)$ of interactions (fixed at 0.5 for all species so that the interaction switch is entirely determined by the functional response to species abundance) while the latter indicates no variation in demographic parameters (i.e. the intrinsic growth rate $\left(r_{i}\right)$ and density-dependent coefficient $\left(c_{i}\right)$ are the same for all species and set at 1 in simulations). Evidently, the interaction switch is only driven by demographic variations in the former, while it is mainly caused by the variation of benefits in the latter.

We compiled 48 pollination networks and 33 seed-dispersal networks from published sources (see supplementary Section S1). Using the numbers of plant species and animal species of these real networks and their interaction numbers as model inputs (other parameters are randomly assigned as above), we simulated the model system and tracked interaction matrices over time [at least $500 \times(m+n)$ steps]. To assess model predictions, we compared the nestedness predicted by the model with observations of the 81 real networks. The nestedness of interaction matrices was measured based on overlap and decreasing fill (NODF; Almeida-Neto et al. 2008) using the software package AnINHADO 3.0 (Guimarães \& Guimarães 2006). The NODF measure of nestedness has a stronger discriminative power of random matrices than other measures and was thus favoured (Ulrich et al. 2009). Moreover, we considered relative nestedness, defined as $\mathrm{NODF}_{\mathrm{R}}=(\mathrm{NODF}-\mathrm{NODF} *) /$ NODF* (Bascompte et al. 2003), where NODF* is the average NODF of 1000 interaction matrices generated randomly by null model Er or Ce provided by AnINHADO 3.0. Reduced major axis regression was used to assess the model performance on predicting the nestedness of real networks (RMA v. 1.17; Bohonak 2004).

\section{RESULTS}

In our theoretical model, the random networks rapidly converged to a nested architecture via the interaction switch, with the nestedness of the mutualistic network stabilised at a certain level (Fig. 1). Similar results were observed when using $\mathrm{NODF}_{\mathrm{R}}$ (Fig. 1f; see supplementary Section S3). Critically, the predicted nestedness concurred precisely with values from real, observed networks (e.g. Fig. 1a-f) regardless of the initial network structures (see supplementary Section S4), whereas models with benefit-neutral interactions (Fig. 1g) and with demography-neutral interactions (Fig. 1h) over- and under-estimated nestedness, respectively.

Overall, the predictions of NODF agreed well with observations (reduced major axis regression: slope $=0.726$ with nearly $80 \%$ variance explained $\left(R^{2}=0.768\right)$; Fig. $\left.2 \mathrm{a}\right)$. The same is true when using $\mathrm{NODF}_{\mathrm{R}}$ (slope $=1.07, R^{2}=0.46$ for the null model Er; slope $=1.06$, $R^{2}=0.40$ for the null model Ce; Fig. 2b), where the $\mathrm{NODF}_{\mathrm{R}}$ was converted into $1 /\left(\mathrm{NODF}_{\mathrm{R}}+1\right)$ due to the negative correlation between relative and absolute nestedness $(r=-0.29, P<0.01$ for the null model Er; $r=-0.23, P=0.04$ for the null model Ce). Moreover, the comparison of model predictions suggests that models with benefit-neutral and demography-neutral interactions produced a higher and lower value of NODF respectively than the general model (Fig. 2c), indicating a negative effect of the variation in species benefits on the nestedness of the mutualistic networks, in contrast to a positive effect of demographic variation on the nestedness.

The number of interactive partners of a species (namely the species degree) was strongly positively correlated with its abundance in our modelled networks, consistent with real observations (Fig. 3a, b). 

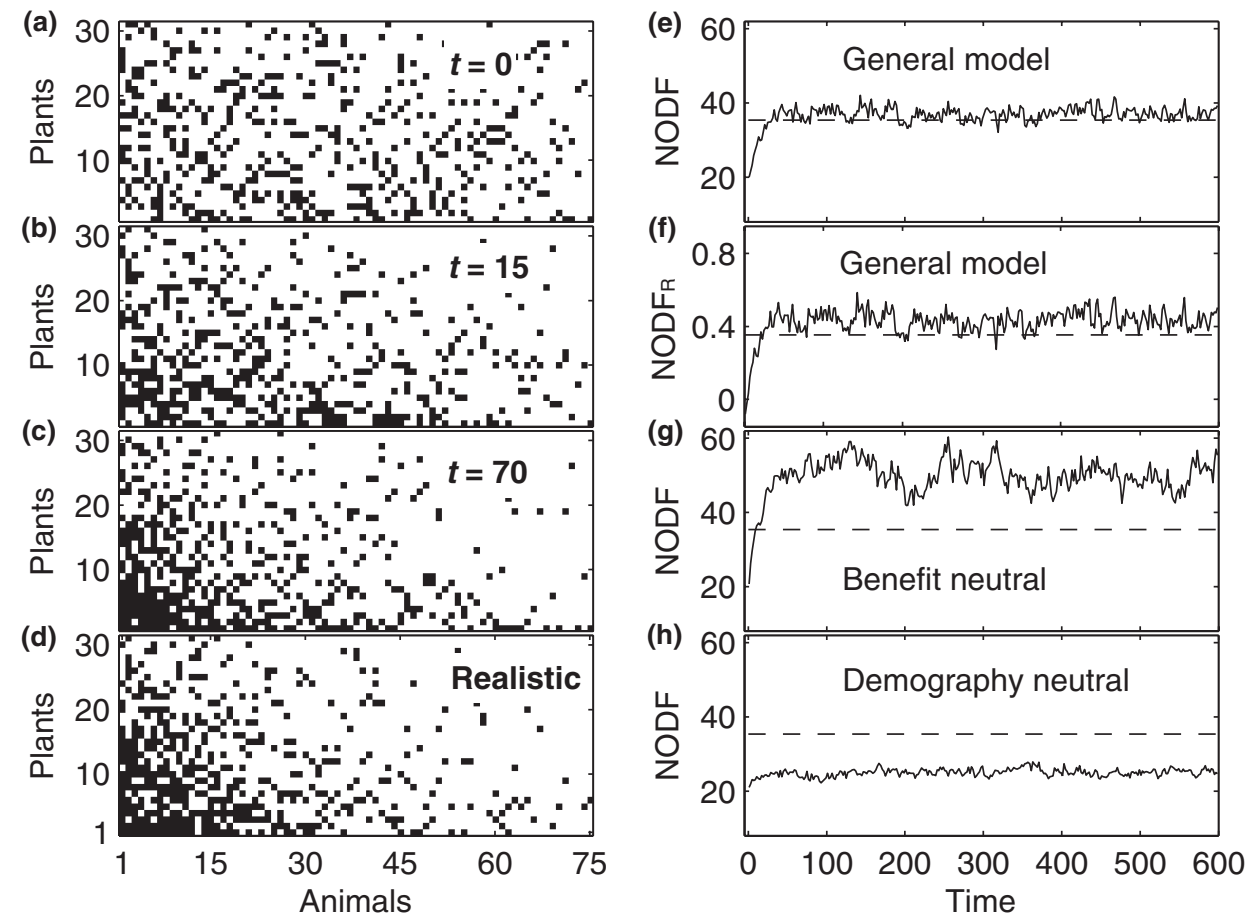

Figure 1 The dynamics of mutualistic interaction networks. Simulations started from a random interaction matrix, which has the same numbers of plant and animal species, as well as the same number of interactions, as the real network of Elberling \& Olesen (P11 in Mutualistic Network Database, see supplementary Section S1). Panel (a), (b) and (c) are the snapshots of interaction matrices at the beginning and time 15 and 70 (note that each time is $m+n$ time steps) respectively. Panel (d) illustrates the interaction matrix of Elberling \& Olesen's real network. Panel (e) and (f) gives the dynamical behaviour of nestedness (NODF) and relative nestedness $\left(\mathrm{NODF}_{\mathrm{R}}\right.$ with the null model Ce provided by Aninhado 3.0) over time for the general model. Panel (g) and (h) gives the dynamics of nestedness for models with only benefit-neutral and demography-neutral interactions respectively. Dashed lines indicate NODF or $\mathrm{NODF}_{\mathrm{R}}$ of the real network. See additional dynamics of absolute and relative nestedness using other null models in ANINHADO 3.0 in Section S3 of the supporting online materials.

Although the benefit matrix was initially randomly assigned, species interact more (i.e. have a higher probability of building an interaction) with the species that can provide a higher benefit in the simulation. Most interactions bring greater mutual benefits to both sides (instead of one species gaining more from its partner than its partner gaining from itself), leading to a high probability of interactions with a trivial difference in benefits for the pairwise interacting species (see supplementary Section S5).

Moreover, once we aligned the simulated species [at time step $200 \times(m+n)]$ according to their abundance ranks in the real network (Fig. 4a, b; i.e. species identity was defined solely by abundance rankings), the degrees of these identified species were found to be consistent with the observed species degrees (Fig. 4a, b). This indirectly suggests that our model could produce accurate speciesspecific predictions. Furthermore, for one specific simulation, without changing the species identity, species degree remained largely unchanged after additional $100 \times(m+n)$ time steps (Fig. 4c, d), suggesting that these species-specific predictions are generally fixed after an initial dynamic re-wiring period. Another indirect test that identified species according to their strength (i.e. the total dependence of other species on them; Bascompte et al. 2006; also see definition in supplementary Section S6) yielded similar results (see supplementary Section S7).

\section{DISCUSSION}

It is clear that species interactions in nature are highly dynamic in both space and time (Whittall \& Hodges 2007; Thompson et al. 2010). Our model considered not only the dynamics of populations but also the active changing of interactions between species over time and demonstrated that a mutualistic network can, from an initially random network, dynamically converge upon the nested architecture with a stable level of nestedness (Fig. 1). Besides predicting the observed nestedness of real networks well, the model can also support other commonly observed patterns in mutualistic networks (see supplementary Section S6): (1) the asymmetry (dissimilarity) between the mutual dependence of plant-animal pairwise interactions (Bascompte et al. 2006; Vázquez et al. 2007), (2) the truncated power-law distribution of species degree (Jordano et al. 2003) and (3) the positive relationship between species degree and species strength (Bascompte et al. 2006). Therefore, these results show that this model has a fundamental robustness and broad applicability in representing mutualistic networks.

One potential reason for this dynamism of species interactions is that species switch their interaction partners to enhance the benefit obtained from another species. The interaction switch in turn may be determined by the new partner's abundance and whether the benefit is mutual or perhaps biased towards one of the interaction partners (Bascompte et al. 2006). An interaction partner is more likely to switch to a more abundant or potentially more beneficial partner species. Because the benefit of an interaction often depends on morphological traits of interacting species (Thompson 2005; Pauw et al. 2009), our results support the hypothesis that species abundance and adaptive morphological traits are important factors in the architecture of mutualistic networks (Ollerton et al. 2003; Rezende et al. 2007; Vázquez et al. 2009). For instance, abundant pollinators have a higher 

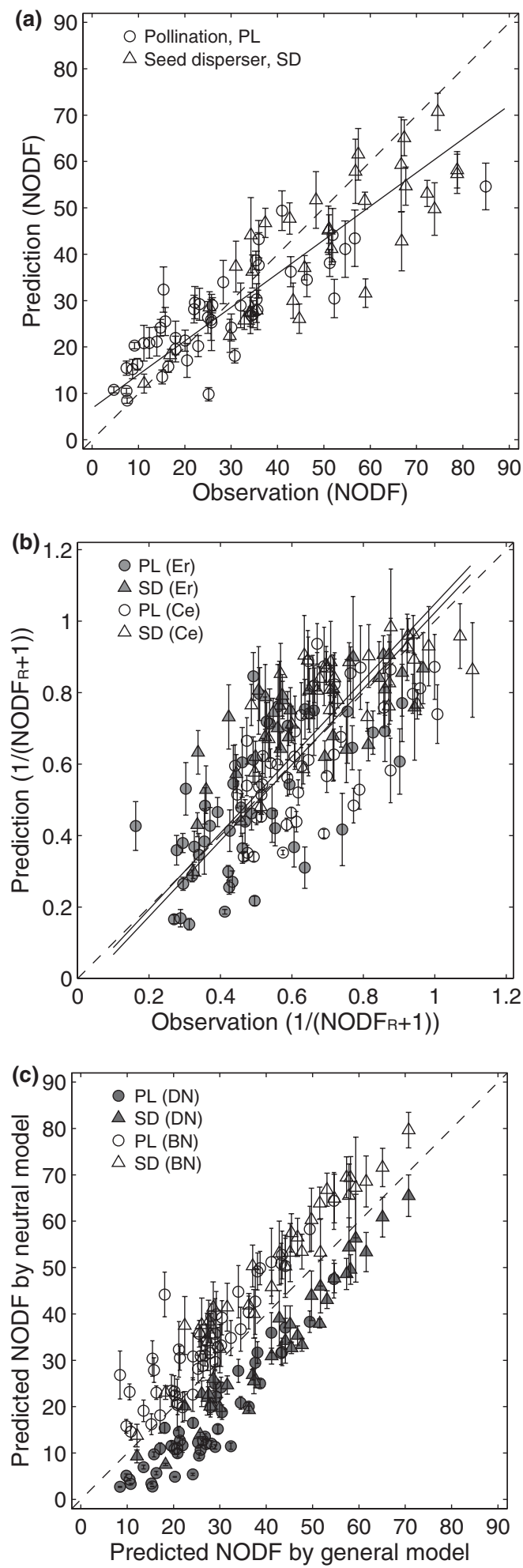

Figure 2 The predicted nestedness of 48 pollination (PL) and 33 seed-dispersal (SD) networks. (a) NODF predicted by the general interaction switch model vs. observations of real networks. (b) $\mathrm{NODF}_{\mathrm{R}}$ [transformed to $1 /\left(\mathrm{NODF}_{\mathrm{R}}+1\right)$ ] predicted by the general model vs. observations of real networks. (c) Predictions from the general model vs. predictions from the model with benefit-neutral (BN) and with demography-neutral (DN) interactions. Predictions are the averages of 300 samplings each $m+n$ time steps after $200 \times(m+n)$ time steps in the simulations. Error bar indicates the standard deviation. The solid lines represent regression results using the reduced major axis and the dashed lines indicate perfect agreement between predictions and observations.
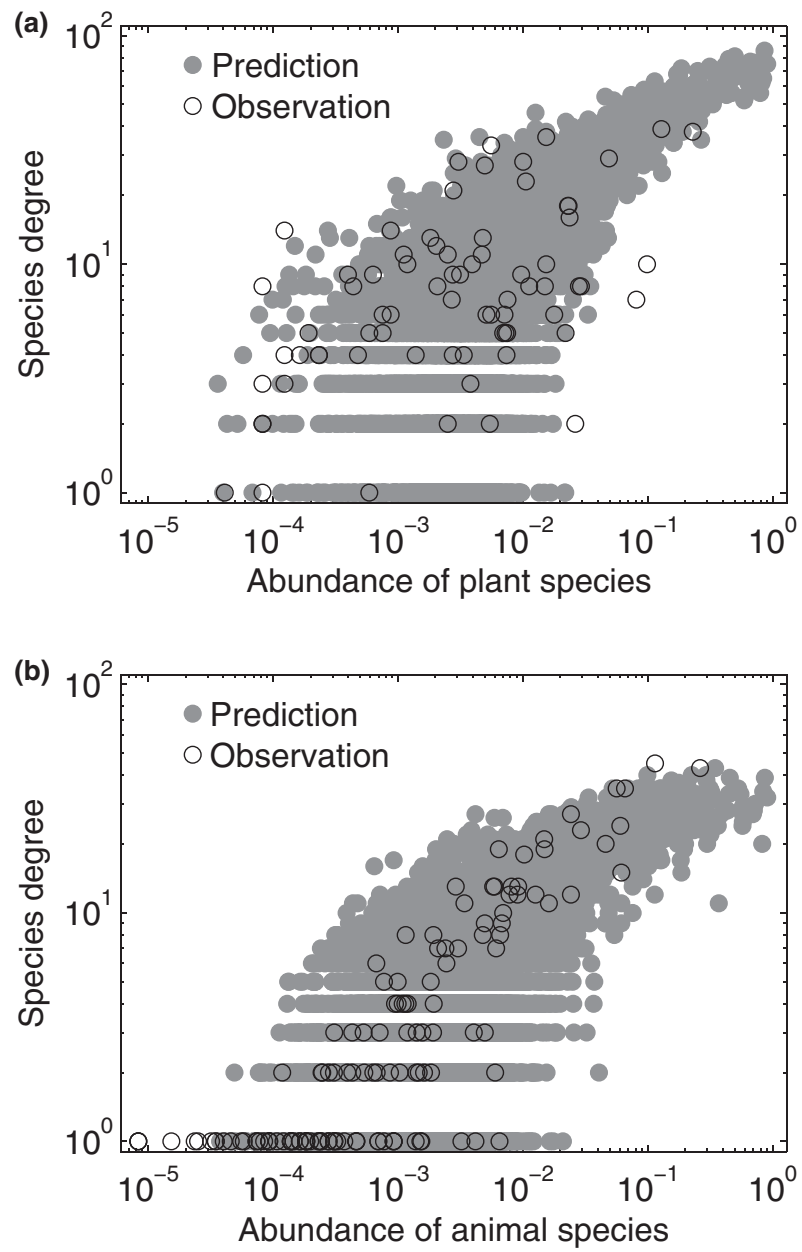

Figure 3 The relationship between species degree (the number of interactions with the focal species) and abundance. Observations are from the restored pollination community of Kaiser-Bunbury et al. (2009) (P18 in Section S1); abundance is depicted by the relative proportion of the plant species (a) or animal species (b). The predictions are snapshots at time step $200 \times(m+n)$ for 100 repeats, with the initial population size, interaction and benefits matrices randomly assigned.

probability of interacting with a wide range of plant species than rare pollinators; similarly, abundant plants are more likely to be visited by a wide range of pollinator species than rare plants (Ollerton et al. 2003; Olesen et al. 2008). Furthermore, morphological traits in mutualistic networks often show evidence of phenotypic complementarity and convergence (Rezende et al. 2007) and constraint interaction (Jordano et al. 2003). Both species abundances and morphological traits produce asymmetric specialisation (Vázquez \& Aizen 2004; Stang et al. 2006, 2007; Anderson et al. 2010) and is a key character of nested architecture (Nielsen \& Bascompte 2007; Krishna et al. 2008).

Moreover, our results clarified the role of species abundance and beneficial interactions in the formation of nested architecture in mutualistic networks (and also in antagonistic networks; see supplementary Section S8). Benefit-neutral interactions often lead to higher predictions of nestedness than predicted from the general model in contrast to the negative effect of demography-neutral interactions on nestedness (Fig. 1e-h and 2c). Krishna et al. (2008) examined a neutral-niche model where species from two neutral communities interact in the network according to the probability of individuals from pairwise species encountering one another. The 

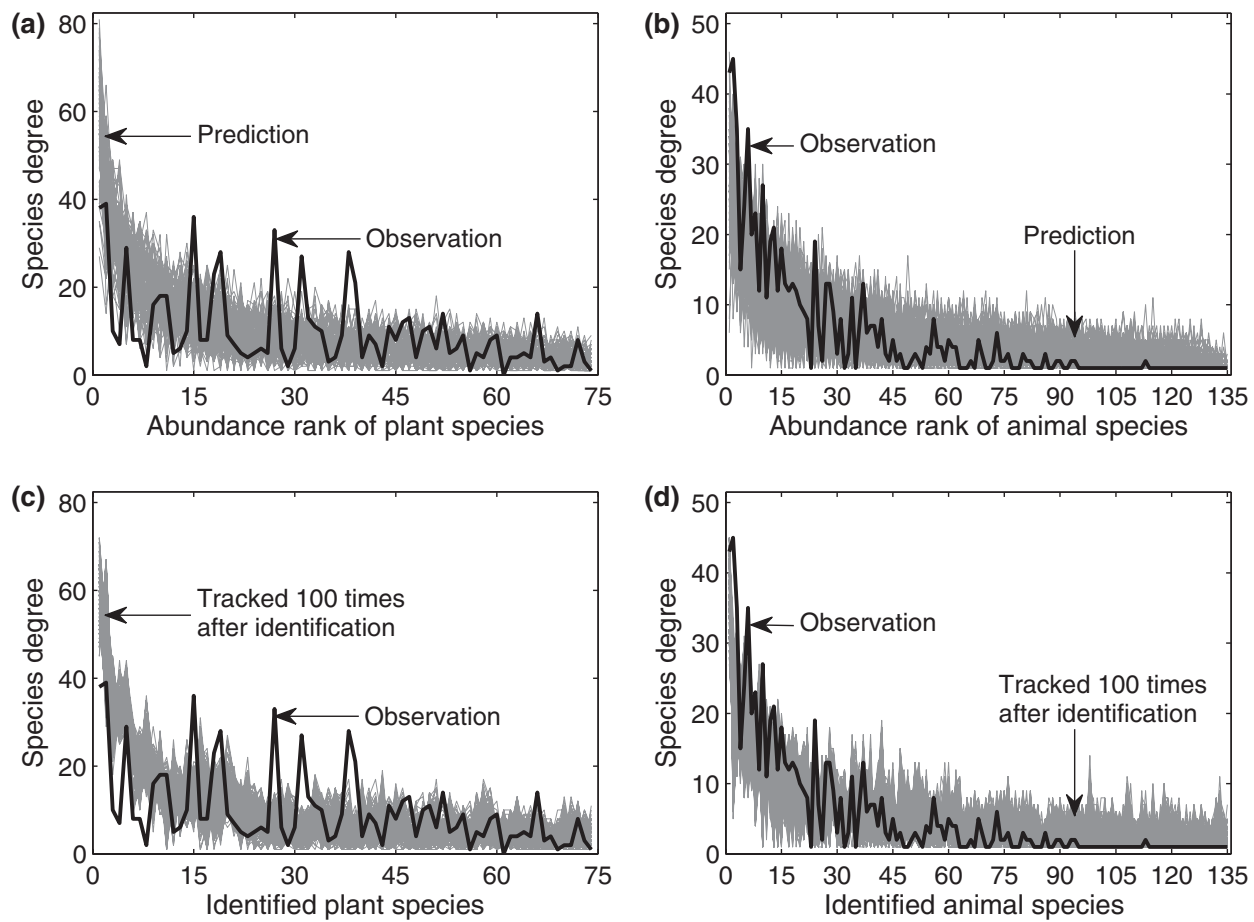

Figure 4 Rank-curves of species degrees. The identities of plant and animal species are assigned according to their observed abundance rankings at time step $200 \times(m+n)$ (a and b). Grey belts are predictions of these species from 100 simulations. For a specific simulation, with species identities held constant, species degrees remain consistent with the observations even after extending the simulation for extra $100 \times(m+n)$ time steps (c and d). Observations are from the restored pollination community of KaiserBunbury et al. (2009) (P18 in Section S1).

neutral-niche model yielded a nearly perfectly nested network and thus overestimates actual nestedness in real networks. Comparing Krishna et al.'s (2008) model prediction with our results from benefit-neutral interactions, we suggest that (1) realistic species-abundance distribution is a key promoter of network architecture and (2) the strength of species interaction is compromised by functional responses in real networks (Holland et al. 2006), rather than a direct result of interactive partner species encountering each other.

A species' ability to switch interaction partners to enhance the relative benefit can give rise to the nested architecture of mutualistic networks. Importantly, the interaction switch, as an adaptive behaviour, allows the nestedness of mutualistic networks to converge towards an intrinsic stable level that mainly depends on network size and the number of interactions in the model. It is important to note, however, that the interaction switch (or other potential adaptive behaviours) only provides the means for an initially random network to approach the stable level of the nested architecture. Regardless, it is striking that such a simple 'rule' allows the model to closely mimic natural, observed networks so well. Indeed, the 81 real networks we tested also stabilised around their known level of nestedness (Fig. 2a, b). Furthermore, interaction switches are also common in food webs (Murdoch 1969; Staniczenko et al. 2010). An antagonistic model incorporating an interaction switch can also lead to nestedness of bipartite food webs (Thébault \& Fontaine 2010), consistent with real networks (see supplementary Section S8). This suggests that the interaction switch could be a prevalent process for the formation of nested architecture in other types of bipartite ecological networks.

Interaction switching can have a profound influence on the complexity-stability relationship in ecological networks (Montoya et al. 2006). By incorporating the interaction switch (i.e. with foraging adaptation), Kondoh (2003) showed that the classic negative complexity-stability relationship in static food webs (May 1972) does not necessarily hold. Beckerman et al. (2006) and Petchey et al. (2008) were able to further predict food-web complexity (connectivity) using interaction switches. In mutualistic networks, allowing interaction switches can enhance the robustedness of interaction strength against species loss over static networks (Kaiser-Bunbury et al. 2010). Thébault \& Fontaine (2010) further demonstrated that a highly connected and nested architecture promotes stability in mutualistic networks. Moreover, Okuyama \& Holland (2008) found that the resilience of mutualistic networks can be enhanced by increasing network size and the number of interactions and, thus, support a positive complexity-stability relationship. Specifically, they reported a weak effect of enhancing resilience by the nested architecture (based on the index matrix temperature; Atmar \& Paterson 1993). Our results suggest that the interaction switch can increase the nestedness of a random network and thus could potentially enhance the stability of mutualistic networks (Okuyama \& Holland 2008; Thébault \& Fontaine 2010). Therefore, interaction switches could be an important process allowing a static community to become a dynamic (or adaptive) one and further foster ecological complexity and diversity.

In conclusion, the nested architecture in mutualistic networks is simply a necessary outcome of adaptive networks (in this case the result of the adaptive behaviour of an interactive switch). We suggest this could be true for any networks with the ability to readjust the links between nodes (species). The results of this study are of fundamental importance to studies of species interactions and ecological networks (Guimarães et al. 2007b; Burgos et al. 2008) and have direct 
implications for understanding network robustness under environmental or anthropogenic perturbations (Kaiser-Bunbury et al. 2009, 2010; Staniczenko et al. 2010).

\section{ACKNOWLEDGEMENTS}

We are grateful to Marcel Holyoak, Jennifer Dunne and four anonymous referees for their constructive suggestions on an earlier version of this manuscript. F.Z. is supported by the DST-NRF Centre of Excellence for Invasion Biology at Stellenbosch University; C.H. is supported by the NRF Blue Sky Research Programme; J.S.T. is supported by the South African National Research Foundation.

\section{AUTHORSHIP}

FZ and $\mathrm{CH}$ designed research; JST collected data; FZ and $\mathrm{CH}$ performed modelling work and analysed data; FZ wrote the first draft of the manuscript and all authors contributed substantially to revisions.

\section{REFERENCES}

Aizen, M.A. \& Vázquez, D.P. (2006). Flowering phonologies of hummingbird plants from the temperate forest of southern South America: is there evidence of competitive displacement? Ecography, 29, 357-366.

Almeida-Neto, M., Guimarães, P., Guimarães, P.R. Jr, Loyola, R.D. \& Ulrich, W. (2008). A consistent metric for nestedness analysis in ecological systems: reconciling concept and measurement. Oikos, 117, 1227-1239.

Anderson, B., Terblanche, J.S. \& Ellis, A.G. (2010). Predictable patterns of trait mismatches between interacting plants and insects. BMC Evol. Biol., 10, 204.

Ascher, U.M. \& Petzold, L.R. (1998). Computer Methods for Ordinary Differential Equations and Differential-algebraic Equations. Society for Industrial and Applied Mathematics, Philadelphia, PA.

Atmar, W. \& Paterson, B.D. (1993). The measure of order and disorder in the distribution of species in fragmented habitats. Oecologia, 96, 373-382.

Barrett, S.C.H. (2010). Darwin's legacy: the forms, function and sexual diversity of flowers. Phil. Trans. R. Soc. B, 365, 351-368.

Bascompte, J. \& Jordano, P. (2007). Plant-animal mutualistic networks: the architecture of biodiversity. Annu. Rev. Ecol. Evol. Syst., 38, 567-593.

Bascompte, J., Jordano, P., Melián, C.J. \& Olesen, J.M. (2003). The nested assembly of plant-animal mutualistic networks. Proc. Natl Acad. Sci. USA, 100, 9383-9387.

Bascompte, J., Jordano, P. \& Olesen, J.M. (2006). Asymmetric coevolutionary networks facilitate biodiversity maintenance. Science, 312, 431-433.

Basilio, A.M., Medan, D., Torretta, J.P. \& Bartoloni, N.J. (2006). A year-long plantpollinator network. Austral. Ecol, 31, 975-983.

Bastolla, U., Fortuna, M.A., Pascual-García, A., Ferrera, A., Luque, B. \& Bascompte, J. (2009). The architecture of mutualistic networks minimizes competition and increases biodiversity. Nature, 458, 1018-1020.

Beckerman, A.P., Petchey, O.L. \& Warren, P.H. (2006). Foraging biology predicts food web complexity. Proc. Natl Acad. Sci. USA, 103, 13745-13749.

Bohonak, A.J. (2004). RMA: Software for Reduced Major Axis regression v. 1.17. Available at: http://www.bio.sdsu.edu/pub/andy/RMA.html. Last accessed 2 March 2011.

Burgos, E., Ceva, H., Hernandez, L., Perazzo, R.P.J., Devoto, M. \& Medan, D. (2008). Two classes of bipartite networks: nested biological and social systems. Phys. Rev. E, 78, 046113.

Chamberlain, S.A., Kilpatrick, J.K. \& Holland, J.N. (2010). Do extrafloral nectar resources, abundances, and body sizes contribute to the structure of ant-plant mutualistic networks? Oecologia, 164, 741-750.

Fortuna, M.A. \& Bascompte, J. (2006). Habitat loss and the structure of plantanimal mutualistic networks. Ecol. Lett., 9, 278-283.

Garcia-Domingo, J.H. \& Saldana, J. (2007). Food-web complexity emerging from ecological dynamics on adaptive networks. J. Theor. Biol., 247, 819-826.
Guimarães, P.R. Jr \& Guimarães, P. (2006). Improving the analyses of nestedness for large sets of matrices. Environ. Model. Soft., 21, 1512-1513.

Guimarães, P.R. Jr, Rico-Gray, V., dos Reis, S.F. \& Thompson, J.N. (2006). Asymmetries in specialization in ant-plant mutualistic networks. Proc. $R$. Soc. London B, 273, 2041-2047.

Guimarães, P.R.Jr, Rico-Gray, V., Oliveira, P.S., Izzo, T.J., Reis, S.F. \& Thompson, J.N. (2007a). Interaction intimacy affects structure and coevolutionary dynamics in mutualistic networks. Curr. Biol., 17, 1-7.

Guimarães, P.R. Jr, Sazima, C., Furtado dos Reis, S. \& Sazima, I. (2007b). The nested structure of marine cleaning symbiosis: is it like flowers and bees? Biol. Lett., 3, 51-54.

Holland, J.N. \& DeAngelis, D.L. (2010). A consumer-resource approach to the density-dependent population dynamics of mutualism. Ecology, 91, 1286-1295.

Holland, J.N., Okuyama, T. \& DeAngelis, D.L. (2006). Comment on 'Asymmetric Coevolutionary Networks Facilitate Biodiversity Maintenance'. Science, 313, 1887.

Holling, C.S. (1959). Some characteristics of simple types of predation and parasitism. Canad. Entomol., 91, 385-398.

Jordano, P., Bascompte, J. \& Olesen, J.M. (2003). Invariant properties in coevolutionary networks of plant-animal interactions. Ecol. Lett., 6, 69-81.

Kaiser-Bunbury, C.N., Memmott, J. \& Müller, C.B. (2009). Community structure of pollination webs of Mauritian heathland habitats. Perspect. Plant Ecol. Evol. Syst., 11, 241-254.

Kaiser-Bunbury, C.N., Muff, S., Memmott, J., Müller, C.B. \& Caflisch, A. (2010). The robustness of pollination networks to the loss of species and interactions: a quantitative approach incorporating pollinator behaviour. Ecol. Lett., 13, 442-452.

Kondoh, M. (2003). Foraging adaptation and the relationship between food-web complexity and stability. Science, 299, 1388-1391.

Krishna, A., Guimarães, P.R. Jr, Jordano, P. \& Bascompte, J. (2008). A neutralniche theory of nestedness in mutualistic networks. Oikos, 117, 1609-1618.

May, R.M. (1972). Will a large complex system be stable? Nature, 238, 413-414.

Montoya, J.M., Pimm, S.L. \& Solé, R.V. (2006). Ecological networks and their fragility. Nature, 442, 259-264.

Murdoch, W.W. (1969). Switching in general predators: experiments on predator specificity and stability of prey populations. Ecol. Monogr., 39, 335-354.

Nielsen, A. \& Bascompte, J. (2007). Ecological networks, nestedness and sampling effort. J. Ecol., 95, 1134-1141.

Okuyama, T. \& Holland, J.N. (2008). Network structural properties mediate the stability of mutualistic communities. Ecol. Lett., 11, 208-216.

Olesen, J.M., Bascompte, J., Elberling, H. \& Jordano, P. (2008). Temporal dynamics in a pollination network. Ecology, 89, 1573-1582.

Ollerton, J., Johnson, S.D., Cranmer, L. \& Kellie, S. (2003). The pollination ecology of an assemblage of grassland asclepiads in South Africa. Ann. Bot., 92, 807-834.

Pauw, A., Stofberg, J. \& Waterman, R.J. (2009). Flies and flowers in Darwin's race. Evolution, 63, 268-279.

Petanidou, T., Kallimanis, A.S., Tzanopoulos, J., Sgardelis, S.P. \& Pantis, J.D. (2008). Long-term observation of a pollination network: fluctuation in species and interactions, relative invariance of network structure and implications for estimates of specialization. Ecol. Lett., 11, 564-575.

Petchey, O.L., Beckerman, A.P., Riede, J.O. \& Warren, P.H. (2008). Size, foraging, and food web structure. Proc. Natl Acad. Sci. USA, 105, 4191-4196.

Rezende, E.L., Lavabre, J.E., Guimarães, P.R. Jr, Jordano, P. \& Bascompte, J. (2007). Non-random coextinctions in phylogenetically structured mutualistic networks. Nature, 448, 925-928.

Stang, M., Klinkhamer, P.G.L. \& van der Meijden, E. (2006). Size constraints and flower abundance determine the number of interactions in a plant-flower visitor web. Oikos, 112, 111-121.

Stang, M., Klinkhamer, P.G.L. \& van der Meijden, E. (2007). Asymmetric specialization and extinction risk in plant-flower visitor webs: a matter of morphology or abundance? Oecologia, 151, 442-453.

Staniczenko, P.P.A., Lewis, O.T., Jones, N.S. \& Reed-Tsochas, F. (2010). Structural dynamics and robustness of food webs. Ecol. Lett., 13, 891-899.

Thébault, E. \& Fontaine, C. (2010). Stability of ecological communities and the architecture of mutualistic and trophic networks. Science, 329, 853-856.

Thompson, J.N. (2005). The Geographic Mosaic of Coevolution. Chicago University Press, Chicago.

Thompson, J.N., Laine, A.L. \& Thompson, J.F. (2010). Retention of mutualism in a geographically diverging interaction. Ecol. Lett., 13, 1368-1377. 
Ulrich, W., Almeida-Neto, M. \& Gotelli, N.J. (2009). A consumer's guide to nestedness analysis. Oikos, 118, 3-17.

Vázquez, D.P. \& Aizen, M.A. (2004). Asymmetric specialization: a pervasive feature of plant-pollinator interactions. Ecology, 85, 1251-1257.

Vázquez, D.P., Melián, C.J., Williams, N.M., Blüthgen, N., Krasnov, B.R. \& Poulin, R. (2007). Species abundance and asymmetric interaction strength in ecological networks. Oikos, 116, 1120-1127.

Vázquez, D.P., Blüthgen, N., Cagnolo, L. \& Chacoff, N.P. (2009). Uniting pattern and process in plant-animal mutualistic networks: a review. Ann. Bot., 103, 14451457.

Whittall, J.B. \& Hodges, S.A. (2007). Pollinator shifts drive increasingly long nectar spurs in columbine flowers. Nature, 447, 706-709.

\section{SUPPORTING INFORMATION}

Additional Supporting Information may be found in the online version of this article:

Section S1 Mutualistic Network Database.

Section S2 Holling's type II functional response for mutualistic networks.
Section S3 The dynamics of nestedness and relative nestedness.

Section S4 The effect of initial structures of the interaction matrices on the stable level of nestedness.

Section S5 Probability distribution of interaction benefits.

Section S6 Predicting other patterns in mutualistic networks.

Section S7 Species rank curves predicted from the model.

Section S8 Antagonistic interaction model and results.

As a service to our authors and readers, this journal provides supporting information supplied by the authors. Such materials are peer-reviewed and may be re-organised for online delivery, but are not copy-edited or typeset. Technical support issues arising from supporting information (other than missing files) should be addressed to the authors.

Editor, Jennifer Dunne

Manuscript received 14 March 2011

First decision made 15 April 2011

Manuscript accepted 1 June 2011 